\title{
Electron cloud instability studies in the Beijing Electron Positron Collider
}

\author{
J. Q. Wang, ${ }^{*}$ Z. Y. Guo, Y. D. Liu, Q. Qin, J. Xing, and Z. Zhao \\ Institute of High Energy Physics, CAS, P.O. Box 918, Beijing 100039, People's Republic of China
}

(Received 1 July 2004; published 21 September 2004)

\begin{abstract}
The electron cloud instability (ECI) in the Beijing Electron Positron Collider (BEPC) was studied systematically with experiments and simulations in recent years. Several electron cloud detectors have been installed into the BEPC storage ring to measure the electron yield. The effects of solenoid, the clearing electrode, the chromaticity, and the octupole on ECI have been investigated in BEPC experimentally. To evaluate the ECI in BEPCII, the upgrade project of BEPC, a computer code has been developed to simulate the electron density in the antechamber under different conditions, as well as the progress of the single bunch and coupled-bunch instabilities caused by the electron cloud. The experimental and simulation results, as well as the activities, are reported in this paper.
\end{abstract}

DOI: 10.1103/PhysRevSTAB.7.094401

PACS numbers: 29.20.Dh

\section{INTRODUCTION}

Since the electron cloud instability (ECI) was first observed on the Beijing Electron Positron Collider (BEPC) in 1996 [1], many experiments have been carried out to study the characteristics of the ECI, the generation of electron cloud (EC), and its effect on the beam performance. In the meantime, analyses and simulations on the ECI in the BEPC storage ring were also done [2]. Now BEPC is being upgraded to a two-ring collider, namely, BEPCII, with electron and positron beams circulating in separated rings. Thus ECI may possibly influence the beam performance in the positron ring as observed in KEKB and PEP-II. As an effective way to reduce the EC, an antechamber with TiN coating will be adopted in BEPCII. A computer code has been developed to estimate the EC density in the beam pipe versus different widths of the antechamber and the secondary electron yield. The single and coupled-bunch instabilities due to EC are also simulated. Meanwhile, to get more practical experience for BEPCII, experiments were continued on BEPC in recent years, such as ECI measurement, solenoid effect, and clearing electrodes [a special detector and beam position monitor (BPM) buttons], as well as octupole to cure ECI.

In this paper, the main experimental results are reviewed with focus on EC measurement and restraining methods such as solenoids, clearing electrodes, chromaticity, and octupole. Then the simulation studies about the ECI in BEPCII are introduced sequentially on the EC density and the single and coupled-bunch instabilities. A summary with some discussions on the results is also given.

\section{EXPERIMENTAL STUDIES}

The main parameters of the BEPC for the typical experimental studies are listed in Table I.

*Electronic address: wangjq@mail.ihep.ac.cn
The instrumentations used in the experiments include tune measurement system, BPM system, synchrotron light monitor, spectrum analyzer, streak camera, etc.

\section{A. First stage studies on ECI phenomena}

The first experiment on the ECI was performed in 1996. A typical chart of the vertical betatron sidebands, which were observed on the spectrum analyzer, is shown in Fig. 1, when the positron beam was injected to $9.3 \mathrm{~mA}$ with 160 uniformly distributed bunches, while the similar phenomena were not found in the case of electron beam under the same conditions. So this vertical coupled-bunch oscillation can be attributed to the electron cloud accumulated in the ring with positron beam. We then started observation on the parameter dependence of ECI, such as bunch spacing, beam energy, beam emittance, closed orbit, chromaticity, etc., as the first stage of experimental studies until 1998.

In the experiments, the instability strongly depends on the bunch spacing, as the threshold current of the instability was higher than $40 \mathrm{~mA}$ when the positron bunches were filled by every two rf buckets. The experiment of beam emittance effect on the ECI showed that the smaller the emittance, the higher the threshold current.

The chromaticity can affect the threshold of the instability. At a beam current of $9.6 \mathrm{~mA}$ with 160 bunches, the vertical betatron sidebands disappeared when chromatic-

TABLE I. Parameters of the BEPC for experiment.

\begin{tabular}{lc}
\hline \hline \multicolumn{1}{c}{ Parameter } & BEPC \\
\hline Beam energy $E(\mathrm{GeV})$ & 1.3 \\
Betatron tune $\nu_{x} / \nu_{y}$ & $5.82 / 6.74$ \\
Bunch spacing $L_{\text {sep }}(\mathrm{m})$ & 1.5 \\
Natural emittance $\varepsilon_{x}(\mathrm{~nm} \mathrm{rad})$ & 134 \\
rf frequency $f_{\mathrm{rf}}(\mathrm{MHz})$ & 200 \\
Harmonic number $h$ & 160 \\
Transverse damping time $\tau_{x, y}(\mathrm{~ms})$ & 86 \\
\hline \hline
\end{tabular}




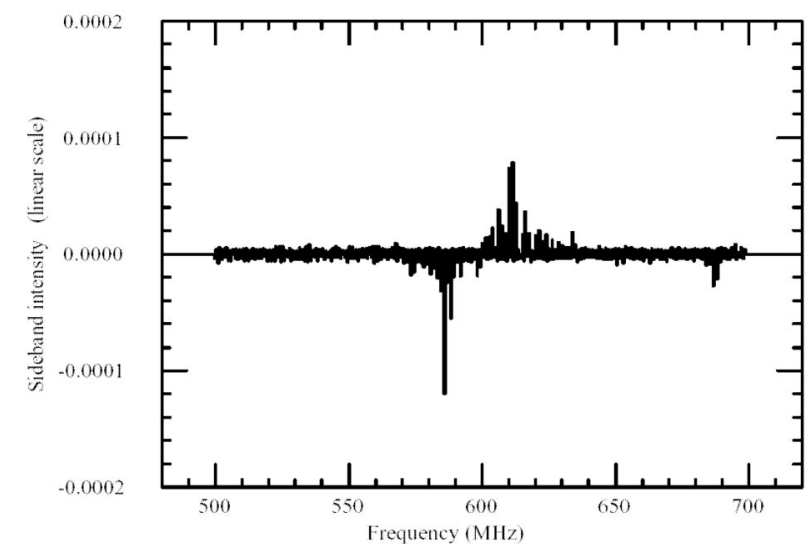

FIG. 1. The vertical betatron sidebands.

ity was tuned from 4 to 6 , and the sidebands reappeared when the chromaticity changed back to 4 . The instability can be suppressed by a larger chromaticity. The energy dependence of the instability is done by scanning from 1.3 to $2.2 \mathrm{GeV}$ at a beam current of $10 \mathrm{~mA}$ with 160 uniformly filled bunches. The observed chromaticity dependence on beam energy at the threshold of the instability is that the higher the beam energy, the lower the chromaticity required to damp the instability, shown in Fig. 2.

The octupole effect on the instability was also tested preliminarily in the first stage experiment. The sidebands of instability could be suppressed when the octupole was excited.

A fast beam position monitor system supplied by KEK was used to study the bunch oscillation process in May 1998. The damping time and the coupled-bunch modes were obtained by fitting and doing fast Fourier transform (FFT) to the oscillation data recorded. This mode analy-

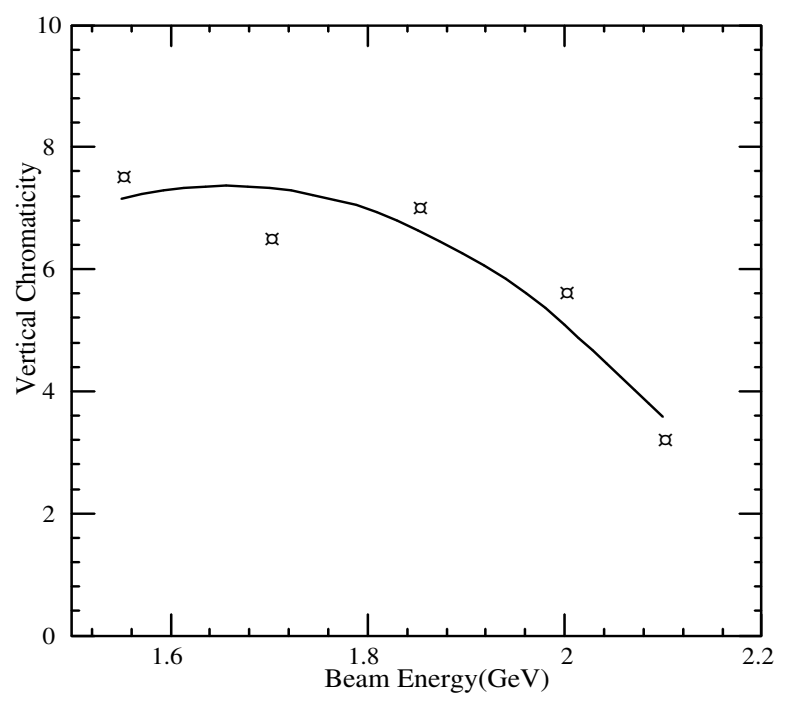

FIG. 2. The chromaticity dependence on beam energy at the threshold of the instability. sis gave a clear picture that the instability is different between positron beam and electron beam under the same conditions.

\section{B. Electron cloud measurement}

EC detectors were installed in the storage ring in 1999. The typical structure of the detector is shown in Fig. 3 with the similar structure as that in the Advanced Photon Source [3]. With the first detector, we got some information on the photon electron (PE) energy distribution and the dependences of beam parameters. From 2002, four other detectors were designed and installed on different positions of the storage ring $[4,5]$.

The detailed EC measurements were carried out using the detectors. The detector current $I_{c}$ varies linearly with the total beam current $I_{b}$, and it did not saturate as the $I_{b}$ was not yet strong enough. The EC energy distribution was deduced from the bias voltage scan. The dependences of $I_{c}$ on beam energy, chromaticity, and emittance are not sensitive at the same closed orbit. The coupled-bunch oscillation caused by ECI does not influence the yield of EC measured.

The multipacting effect has been investigated by filling the positron beam with different bunch spacing, but there is no obvious enhancement of $I_{c} / I_{b}$ by varying the bunch spacing when we used a detector near the magnet. We suspected that the photon electrons were dominant near the exit of the magnet, so another detector was installed far away from the magnet. More experiments are needed to check the multipacting effect.

\section{Solenoid effect on ECI}

It was verified in KEKB [6] and PEP-II [7] that the solenoid field along the beam axis is very effective in suppressing ECI. From the measured $I_{c}$ with the first electron detector when solenoid was wound on the beam pipe nearby, it was clear that the solenoid really affected

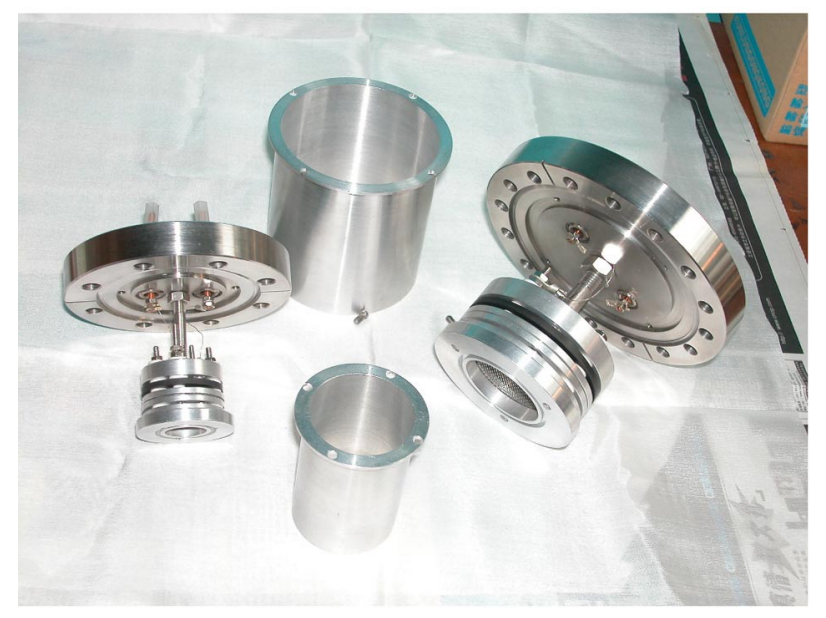

FIG. 3. The detector structure for EC measurement. 

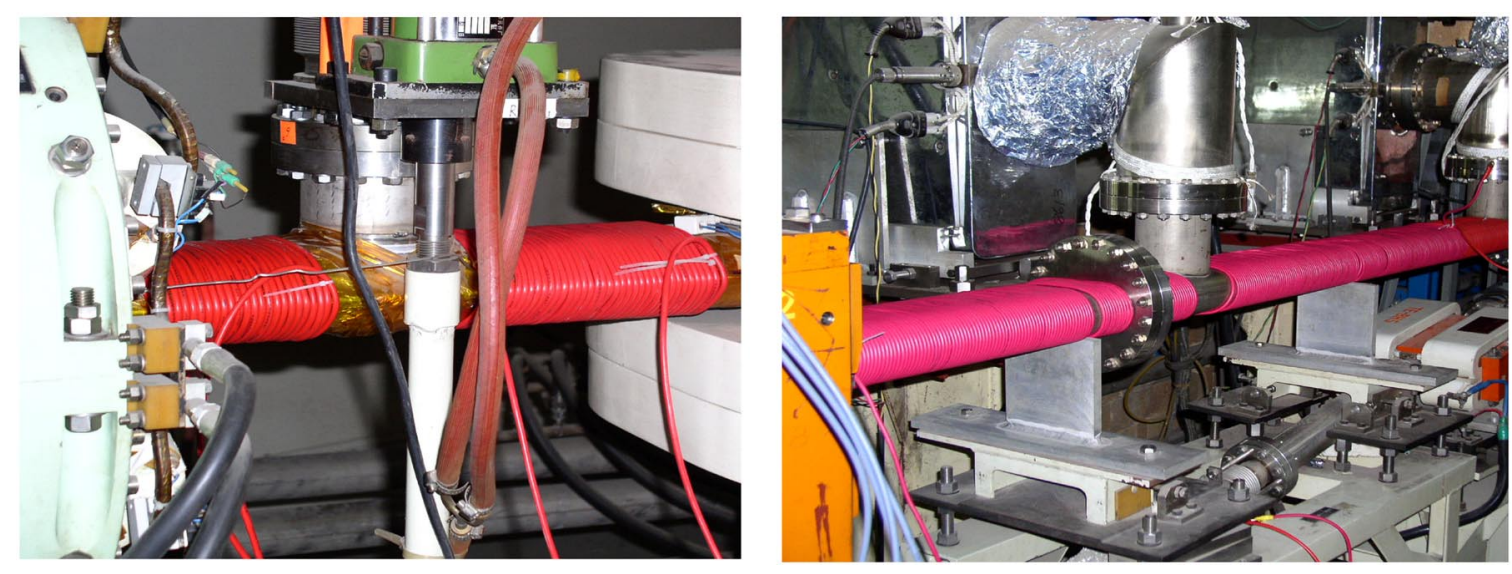

FIG. 4. (Color) The solenoid wound on the vacuum chamber.

the EC density. Thus during the shutdown of BEPC last year, we wound solenoids in most straight sections around the BEPC storage ring to study its effect.

The total length of the solenoid wound on the straight sections is about $42 \mathrm{~m}$, covering about $18 \%$ of the ring circumference. Some pictures of solenoid winding are shown in Fig. 4. All the solenoid parts covered on different straight sections are connected in series. A dc power supply can provide a current up to 35 A to the solenoids, corresponding to about $30 \mathrm{G}$ magnetic field along the beam path.

To study the solenoid effect on coupled-bunch instability, a multibunch positron beam was filled to the threshold current of ECI; we observed clear sidebands of oscillation when chromaticities were set to $\xi_{x, y}=1.5$. When a current of $15 \mathrm{~A}$ was powered to the solenoid, both the left and the right sidebands disappeared, as shown in Fig. 5.

To study the solenoid effect on a single bunch, a streak camera was used to observe the change of the vertical bunch size as the solenoids were powered on and off, respectively. We take the fifth bunch from the tail of the bunch train for estimation. When the solenoids were on, the vertical bunch size was reduced about $15 \%$ on average, as shown in Fig. 6.

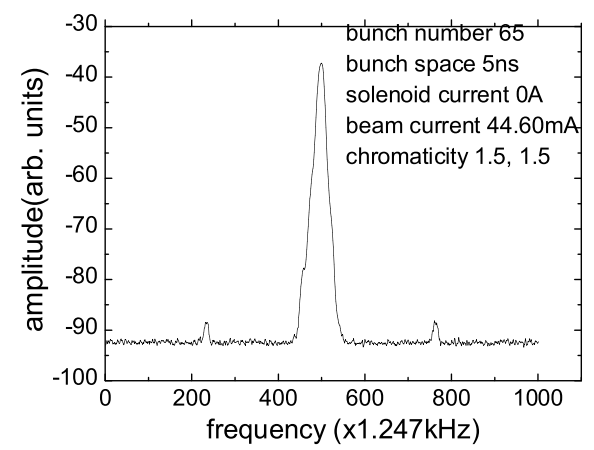

Similar experiments were repeated under different connection schemes of the solenoid parts between the arc and the straight section. From the experiments, it can be seen that the solenoid is more effective in the arc section near the magnet than in the long straight section far away from magnet, and the polarity of the solenoid is not important.

\section{Clearing electrode effect on ECI}

Two kinds of clearing electrode have been tested in the experiment. First, a newly installed EC detector can be used as an electrode to clean the EC with a dc voltage properly given on an extra grid inside, while another detector, which is located just above it on the top, can be used to measure the change of EC after applying the electrode, as shown in Fig. 7. One typical measured electron current $I_{c}$ as a function of the applied voltage is also shown in Fig. 7.

It is observed that the EC density decreases as the voltage applied on the electrode increases, and saturates gradually as the applied voltage is high enough. This result encourages us to do more experiments using the buttons of BPMs as clearing electrodes.

There are 32 BPMs for orbit measurement along the BEPC storage ring. With a special switcher, the total 128

FIG. 5. Solenoid effect on vertical betatron sidebands (left: solenoid off; right: solenoid on).

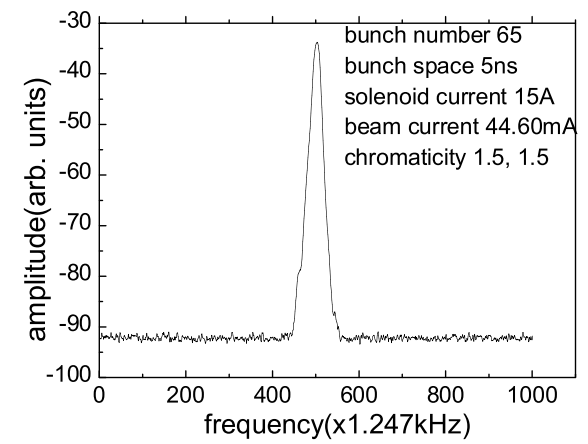



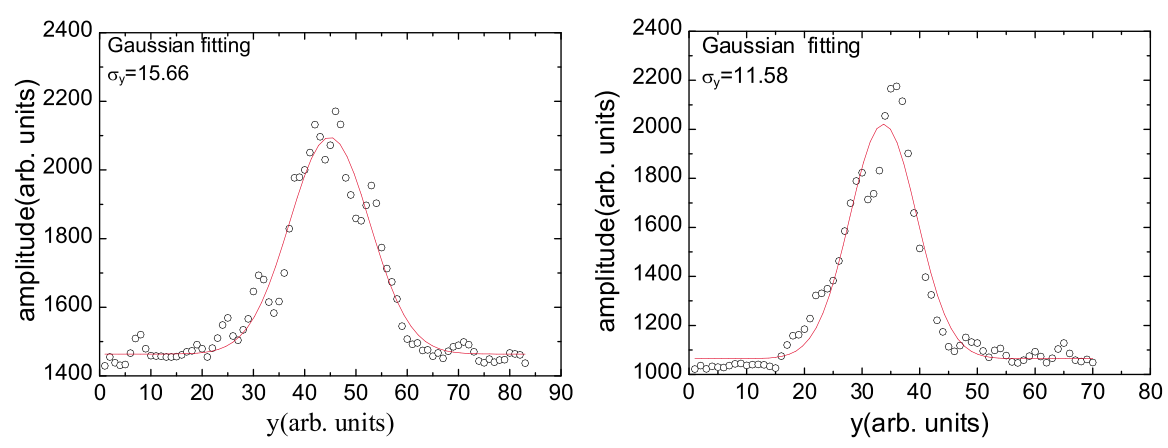

FIG. 6. (Color) The vertical bunch size observed with the streak camera as the solenoid off (left) and on (right).
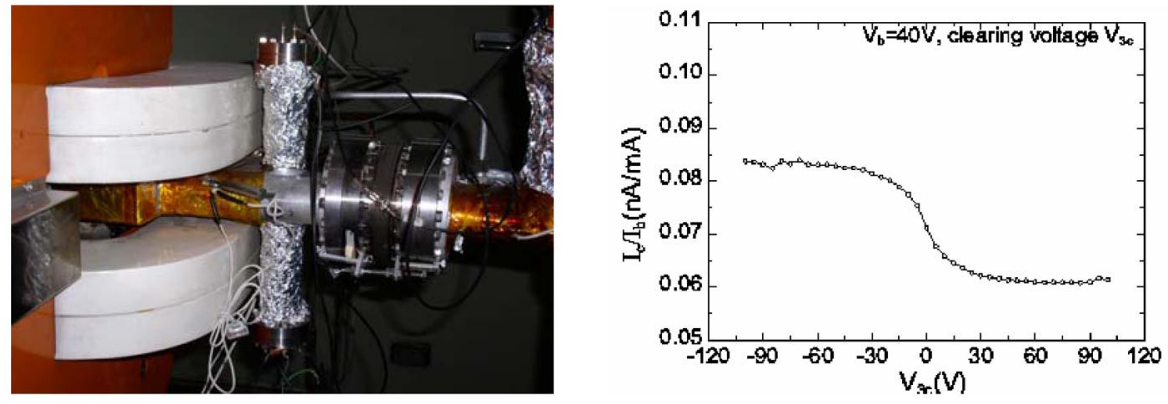

FIG. 7. (Color) The picture of the detectors used as the clearing electrode and the measured $I_{c}$ versus the voltage.

circular BPM buttons can be supplied dc voltage at the same time. In each BPM, the four buttons are labeled as $A, B, C$, and $D$, respectively, as shown in Fig. 8. All the 32 buttons of $A$ or $B$ or $C$ or $D$ can be as different groups to be applied dc voltages. The dc voltages from -600 to $+600 \mathrm{~V}$ can be applied to the buttons with different connection patterns.

At the beam conditions of the ECI, we scanned the applied voltage on the BPM buttons and measured the amplitudes of the observed betatron sidebands. The best pattern is to apply $+600 \mathrm{~V}$ on all $A$ and $D$ buttons, and, in the meantime, apply $-600 \mathrm{~V}$ on all $B$ and $C$ buttons. In this pattern, the amplitudes of the sidebands are $20 \%$ less than the case without any de voltages on the BPM buttons. In this way, the amplitudes of the sidebands and the vertical beam size observed of head bunch and tail bunch

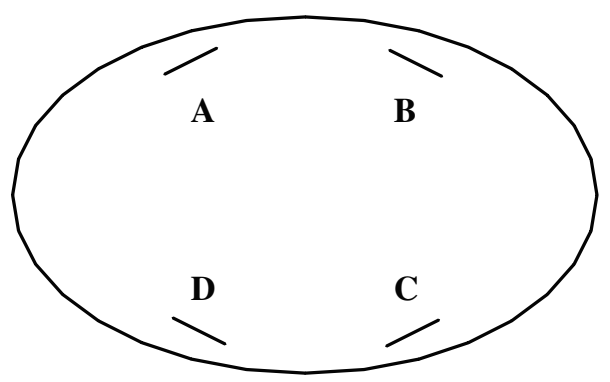

FIG. 8. BPM buttons ( $A, D$ : inboard side of the ring; $B, C$ : outboard side). versus the voltages on the BPM buttons are shown in Fig. 9.

\section{E. Chromaticity effect on ECI}

An effective way to suppress the ECI is to enhance the chromaticity, which had already been verified in BEPC. Higher chromaticity can not only lower the amplitude of sidebands, but it can also reduce the bunch size. On average, the bunch size is reduced about $30 \%$ when the chromaticity is enhanced from 1.5 to 4 in the case of solenoid field off. The amplitudes of sidebands and the vertical bunch size observed of head bunch and tail bunch versus the chromaticity are shown in Fig. 10.

\section{F. Octupole Effect on ECI}

There was one octupole located in the dispersion free straight section of the ring. Its effect against ECI was tested at the threshold beam current of ECI. When the octupole is excited with a dc current of $1.0 \mathrm{~A}$, the vertical sidebands of ECI disappeared and the bunch size of the tail bunch was suppressed about 20\%, shown as Fig. 11. It is expected that the octupole can increase the Landau damping; however, its effect in suppressing the vertical bunch size needs further investigation.

\section{SIMULATION STUDIES FOR BEPCII}

BEPC is being upgraded to a double ring collider with a luminosity aiming at $10^{33} \mathrm{~cm}^{-2} \mathrm{~s}^{-1}$. The ECI is 

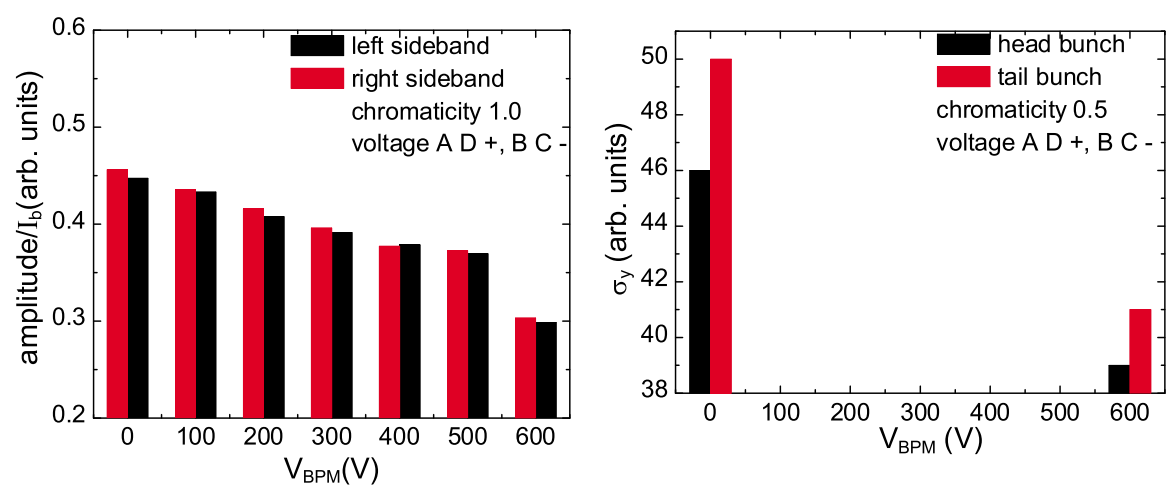

FIG. 9. (Color) Electrode effect on the sideband (left) and the bunch size (right) versus voltage on the BPM buttons.
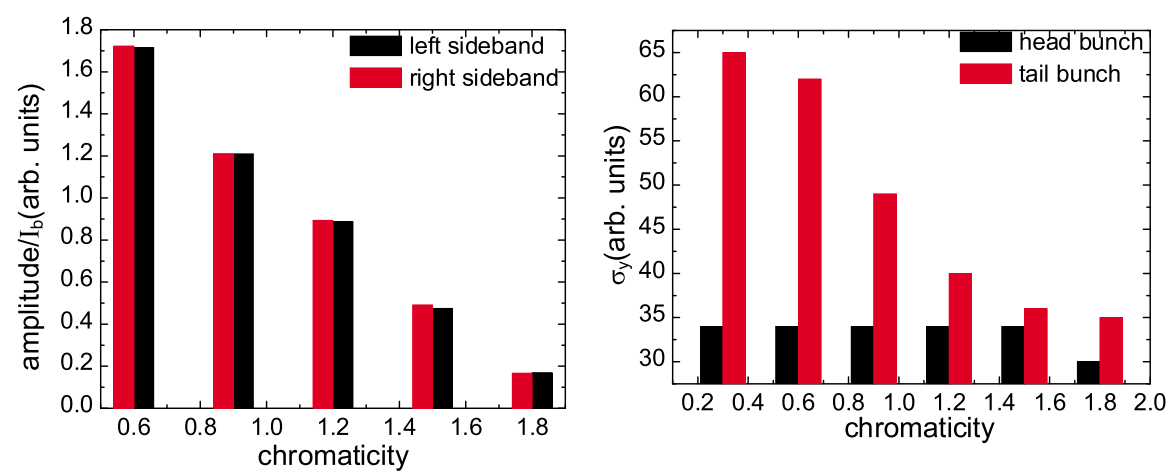

FIG. 10. (Color) Amplitude of the sideband (left) and the bunch size (right) versus chromaticity.
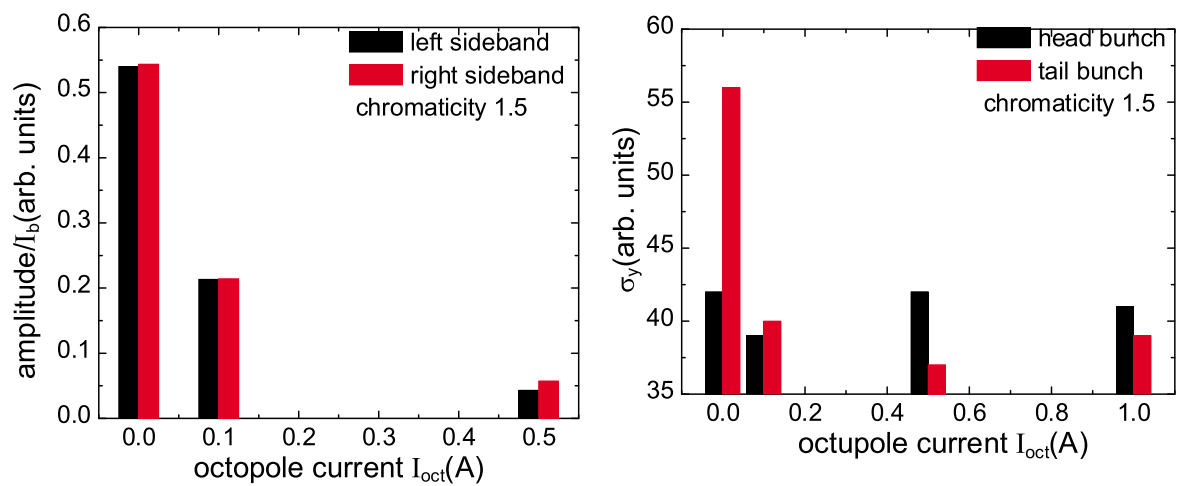

FIG. 11. (Color) Amplitude of the sideband (left) and the bunch size (right) versus octupole strength.

suspected to occur in the positron ring and influence the luminosity performance of the collider, so it has been decided to use an antechamber with an absorber in the arc sections of the ring, and TiN will be coated inside the beam chamber. To evaluate the ECI practically, a code [8] has been developed to simulate the EC density distribution in the antechambers. The coupled-bunch instability and the beam blowup as the head-tail model are also studied with the code. The main parameters relating the ECI are listed in Table II. The typical structure of the antechamber in the arc and the equivalent cross section of the beam pipe used in the simulation are as shown in Fig. 12.

\section{A. Electron cloud}

We consider only two main sources of electrons [9], namely, (i) photoelectrons arising from the synchrotron radiation and hitting the wall of the vacuum chamber and (ii) secondary emission from electrons hitting the walls. Because of the antechamber, only $\sim 0.5 \%$ photons remain inside the beam pipe. Photoelectrons are produced in the 
TABLE II. Parameters of the BEPCII.

\begin{tabular}{lc}
\hline \hline \multicolumn{1}{c}{ Variable } & BEPCII \\
\hline Beam energy $E(\mathrm{GeV})$ & 1.89 \\
Particle population per bunch $N_{b}\left(10^{10}\right)$ & 4.84 \\
Bunch spacing $L_{\text {sep }}(\mathrm{m})$ & 2.4 \\
rms bunch length $\sigma_{z}(\mathrm{~m})$ & 0.015 \\
rms bunch sizes $\sigma_{x, y}(\mathrm{~mm})$ & $1.18,0.15$ \\
Chamber half dimensions $h_{x, y}(\mathrm{~mm})$ & 60,27 \\
Slippage factor $\eta\left(10^{-3}\right)$ & 22 \\
Synchrotron tune $Q_{s}$ & 0.033 \\
Transverse tunes $Q_{x, y}$ & $6.53,7.58$ \\
Circumference $C(\mathrm{~km})$ & 0.24 \\
Average beta function $(\mathrm{m})$ & 10 \\
\hline \hline
\end{tabular}

chamber and antechamber by the photons hitting on the wall with a yield rate of $Y \sim 0.1$ and a reflectivity of $R \sim$ 0.1 . If there is a photon absorber, the $Y$ becomes as small as $Y \sim 0.02$. The percentage of photoelectrons escaping out of the antechamber depends on the ratio of the width of the antechamber to its height, i.e., $L / h$. In the simulation the beam field is presented by the Bassetti-Erskine formula [10] and the numerical solver of POISSONSUPERFISH in the central region of $\left(10 \sigma_{x}, 10 \sigma_{y}\right)$ and out of this region, respectively. In the simulation we assume that the secondary electron yield (SEY) with and without TiN coating in the chamber is 1.06 and 1.8 , respectively.

The line density of EC along the chamber and the volume density of $\mathrm{EC}$ in the central region $(10 \sigma$ of transverse beam size) of the chamber can be obtained from the simulation. Since the central part of the electron cloud is effective in acting on the beam, it is usually counted as EC density in the simulation. The EC distribution in the different shapes of the vacuum chamber and the comparison of that with two electrodes placed near the entrance of the antechamber are shown in Fig. 13. It can be seen that the antechamber and the electrode help to
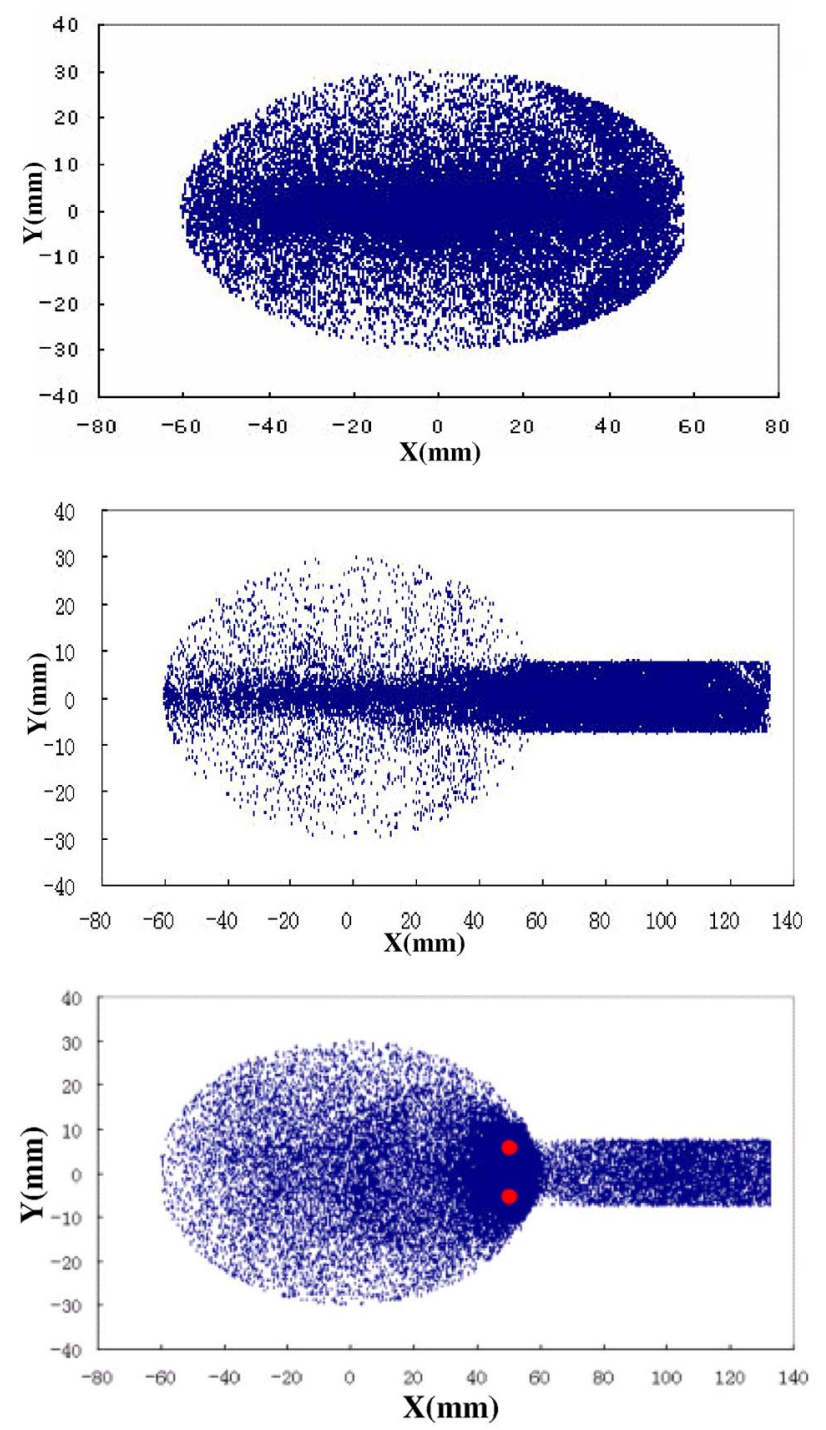

FIG. 13. (Color) The electron cloud distribution in the elliptical chamber (top), in the antechamber (middle), and in the antechamber with electrode (bottom).

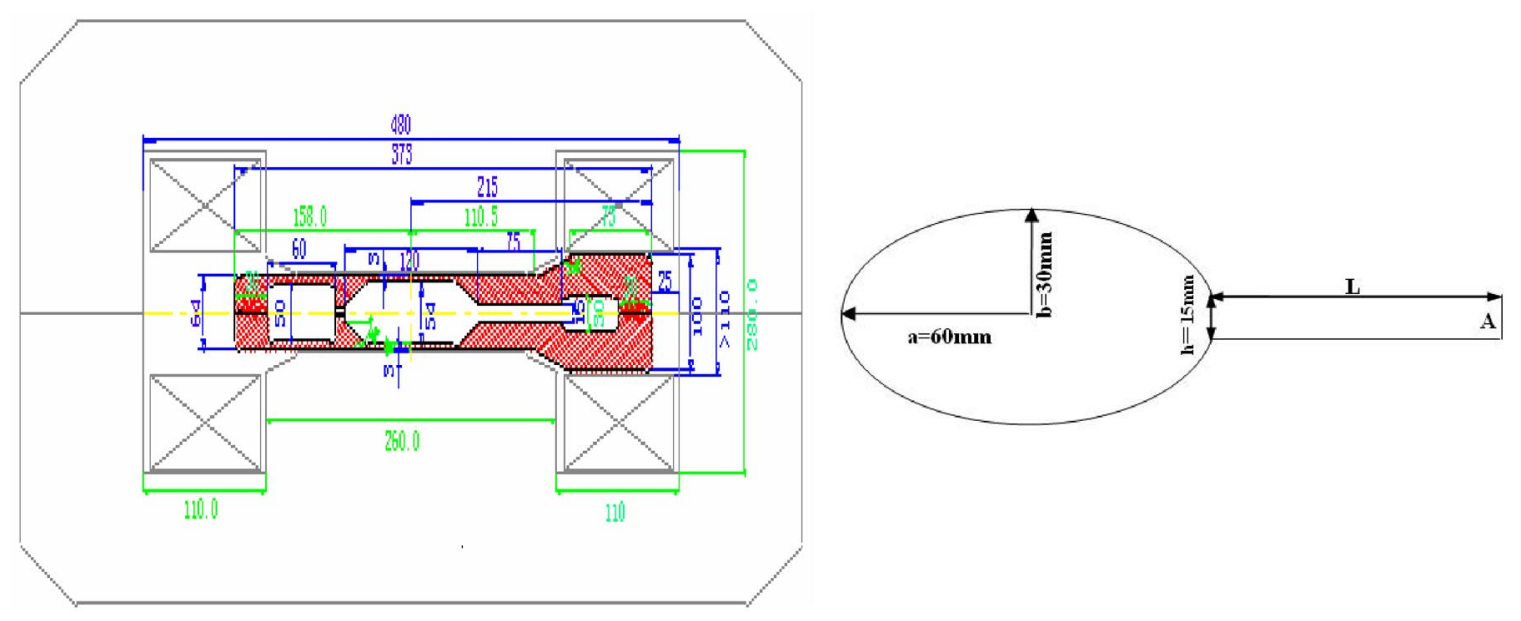

FIG. 12. (Color) The antechamber structure (left) and the simplified model used in the simulation (right). 
reduce the electron density, especially in the central region of the chamber where the beam passes through. The electron density decreases as the voltage on the electrode increases, and the effect will be saturated around $600 \mathrm{~V}$ at the BEPCII parameters.

The EC density accumulated along the bunch train in the antechamber with different $L / h$ is shown in Fig. 14 . The EC density is reduced 5 times if the antechamber width $L$ is about 5 times that of the chamber height $h$. Figure 14 also shows that the electron density will saturate when about 20 to 30 bunches pass, and will dissipate quickly through about ten empty rf buckets.

Simulation with different SEYs shows that the EC density increases quickly when $\delta_{\max }>1.6$, thus TiN coating is necessary for reducing the EC density.

Simulation results show that the EC density can be reduced by about 5 times if the antechamber is adopted, by about 6 times if TiN coating is used only, by about 3 times if a photon absorber is made in the wall of the chamber only, and by about 5 times if an electrode is installed in the beam chamber. In BEPCII, it has been decided to adopt the antechamber, the photon absorber, and the TiN coating. With these three effects being taken into account in the simulation, the electron density will be decreased about 80 times, i.e., from $1.1 \times 10^{13} \mathrm{~m}^{-3}$ in the case without any restraining method to $1.3 \times 10^{11} \mathrm{~m}^{-3}$, which is lower than the threshold causing the strong headtail instability as described in later sections.

\section{B. Single bunch instability}

The electron cloud can act as a short range wake field, and drive single bunch instability. Based on the head-tail model [11], the code also simulates bunch blowup. In the model, transverse distributions of the electron cloud and the bunch are represented by macroparticles. The bunch is longitudinally divided into slices, which interact with the $\mathrm{EC}$ and cause the distortion of the EC distribution. The

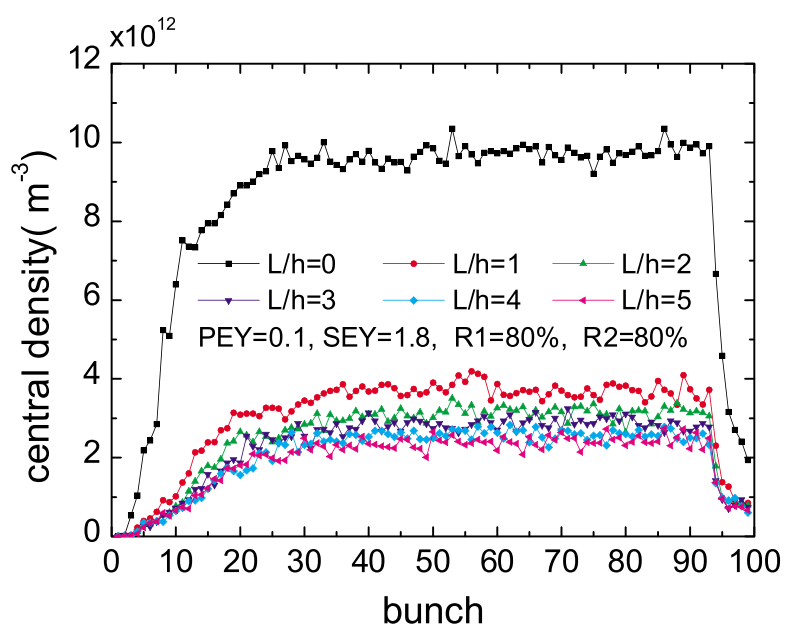

FIG. 14. (Color) EC density versus width of the antechamber. macroparticles in different slices can change their positions due to the synchrotron oscillation. The short range wake field and the bunch size blowup can then be issued from the simulation. Simulation shows that the wake strength is proportional to the EC density, as shown in Fig. 15.

For strong head-tail instability theory, there is a criterion to calculate the threshold. It is expressed as [12]

$$
\Gamma=\frac{N_{b} r_{e}\left|W_{y}(0)\right| \bar{\beta}_{y}}{16 \gamma \nu_{s}} \leq 1
$$

This gives the wake field threshold of $1.47 \times 10^{6} \mathrm{~m}^{-2}$, corresponding to the EC density of about $9.2 \times 10^{11} \mathrm{~m}^{-3}$. Tracking the bunch for 4096 turns with different EC density, we found that the vertical bunch size increases sharply when the EC density is higher than $9.2 \times$ $10^{11} \mathrm{~m}^{-3}$, as shown in Fig. 16. This can be thought of as the threshold of instability and is comparable with the formula.

If the chromaticity is introduced in the simulation, the growth of bunch size becomes saturated and the higher the chromaticity, the stronger the damping effect, as shown in Fig. 17. This result can be compared with the experimental observation of the BEPC.

\section{Coupled-bunch instability}

The coupled-bunch instability may occur in BEPCII as the electron density accumulated along the bunch train. In addition to the simulation of the buildup process of EC density, we track the motion of 93 bunches in a train and record their positions in each turn. The growth time is obtained by fitting the amplitude of the oscillation, and the coupled-bunch mode can be obtained by FFT of the data, as shown in Fig. 18. For BEPCII with the antechamber and TiN coating adopted, the EC density will decrease to $1.35 \times 10^{11} \mathrm{~m}^{-3}$. The growth time of the coupled-bunch instability is about $4.3 \mathrm{~ms}$, which can be damped with the feedback system.

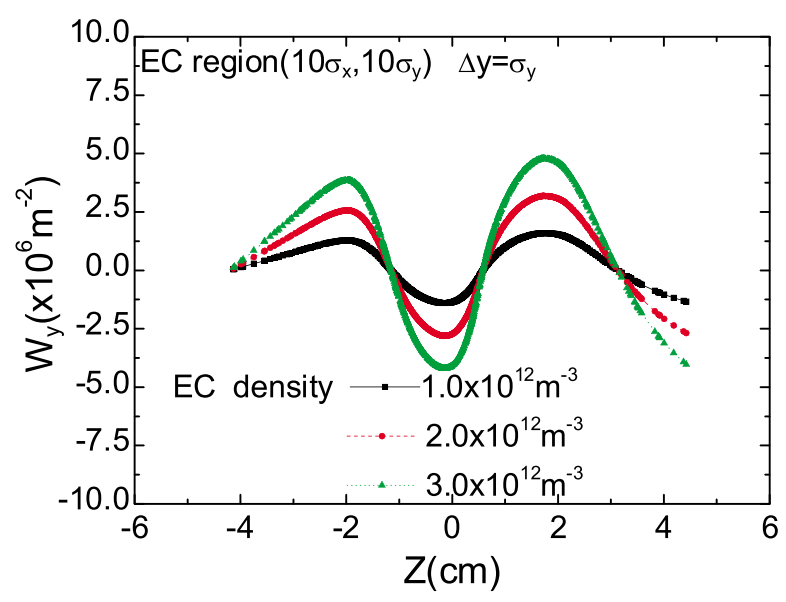

FIG. 15. (Color) Short-range wake due to EC. 

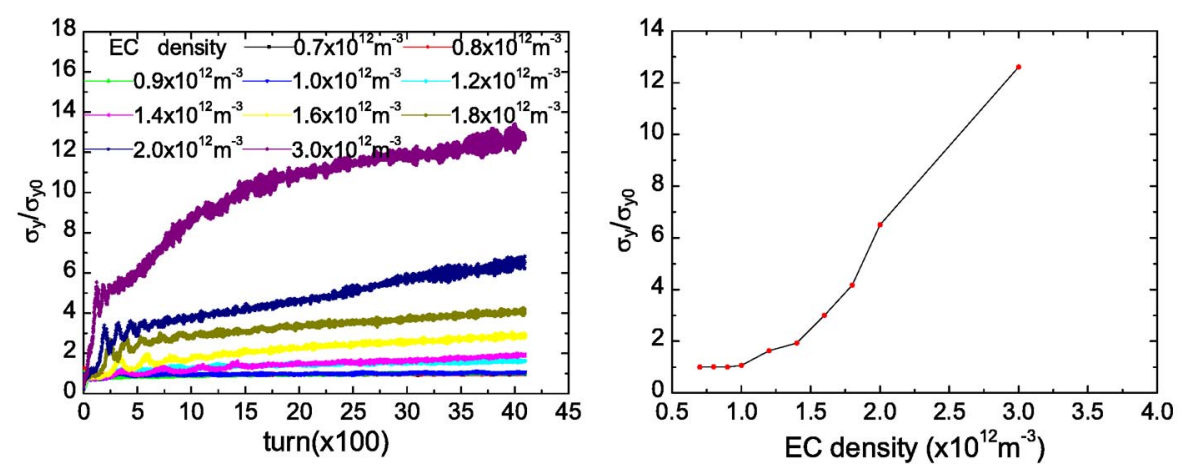

FIG. 16. (Color) Bunch size versus different EC densities.
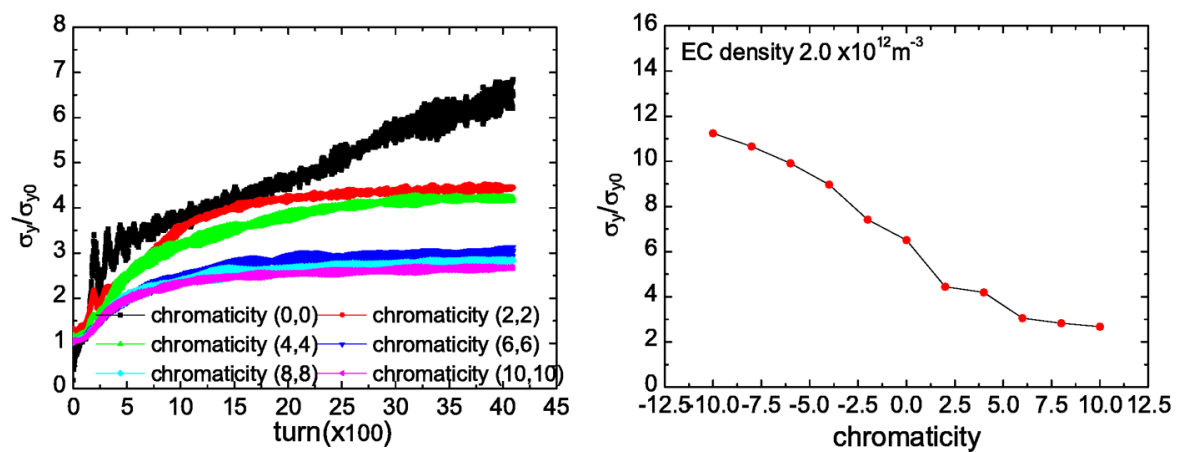

FIG. 17. (Color) The bunch size versus chromaticity.
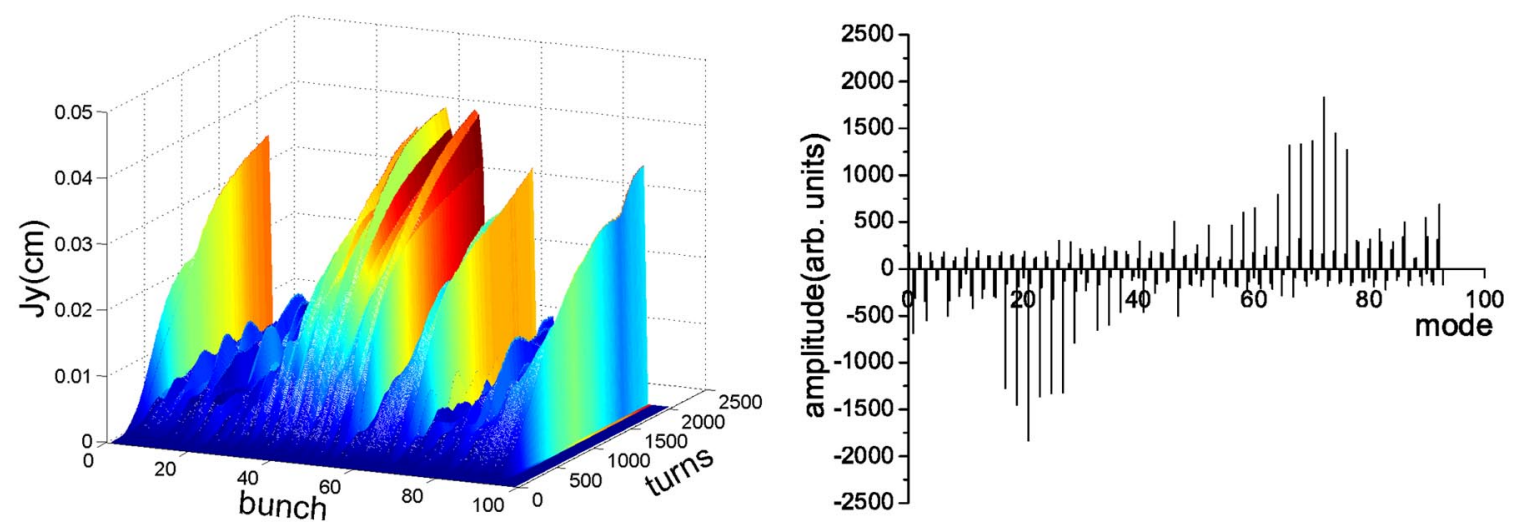

FIG. 18. (Color) Growth behavior of coupled-bunch oscillation and the mode distribution.

\section{SUMMARY}

A long term study on the beam electron cloud instability has been progressing in BEPC for years, and quite rich study results have been obtained from the experiments and the simulations. We have investigated experimentally the effective ways to suppress ECI, such as solenoid winding, clearing electrode, large chromaticity, octupole, etc. And with simulation, the efficiency for the antechamber with photon absorber, TiN coating, and clearing electrode to reduce the EC density is explored.
All of these results are very meaningful for understanding the mechanism for the design and operation of a storage ring for factory-like colliders. Particularly, we have decided to adopt the antechamber with photon absorber and TiN coating in the BEPCII to cure the ECI. The EC density can be suppressed to be below the threshold of strong head-tail-like instability, while the coupled-bunch instability can be damped with the feedback system. However, some of the effects related to ECI still need further study. 


\section{ACKNOWLEDGMENTS}

We thank the BEPC operation team, instrumentation group, and vacuum group for their continuous support of the experiments. The ECI experiments have been well collaborated between IHEP and KEK since 1996. Many discussions with Dr. K. Ohmi and H. Fukuma from KEK are very helpful to the study. This work was supported by the Chinese National Foundation of Natural Sciences, Contracts No. 10275079 and No. 10375076.

[1] Z. Y. Guo, H. Huang, S. P. Li, D. K. Liu, L. Ma, Q. Qin, L. F. Wang, J. Q. Wang, S. H. Wang, C. Zhang, F .Zhou, Y. H. Chin, H. Fukuma, S. Hiramatsu, M. Izawa, T. Kasuga, E. Kikutani, Y. Kobayashi, S. Kurokawa, K .Ohmi, Y. Sato, Y. Suetsugu, M. Tobiyama, K. Yokoya, and X. L. Zhang, in Proceedings of the Particle Accelerator Conference (PAC'97), Vancouver, 1997 (IEEE, Piscataway, NJ, 1997), p. 1566.

[2] Z. Y. Guo, H. Huang, S. P. Li, D. K. Liu, L. Ma, Q. Qin, J. Q. Wang, S. H. Wang, G. Xu, C. Zhang, Z. Zhao, Y. H. Chin, H. Fukuma, S. Hiramatsu, M. Izawa, T. Kasuga, E. Kikutani, Y. Kobayashi, S. Kurokawa, K. Ohmi, Y. Sato, Y. Suetsugu, M. Tobiyama, K. Tsukamoto, K. Yokoya, and X. L. Zhang, Phys. Rev. ST Accel. Beams 5, 124403 (2003).

[3] K. C. Harkay, R. A. Rosenberg, Z. Y. Guo, and Q. Qin, in Proceedings of the Particle Acclerator Conference (PAC01), Chicago, 2001 (IEEE, Piscataway, NJ, 2001), p. 671.

[4] Q. Qin, Z.Y. Guo, H. Huang, Y. D. Liu, J. Q. Wang, J. Xing, Z. Zhao, and H. Fukuma, in Proceedings of the 3rd Asian Particle Accelerator Conference (APAC04), Gyeongju, Korea, 2004 (to be published).
[5] Z. Y. Guo, Y. D. Liu, Q. Qin, J. Q. Wang, and J. Xing, in Proceedings of the Asian Particle Accelerator Conference (APAC04), Gyeongju, Korea, 2004 (Ref. [4]).

[6] H. Fukuma, K. Akai, N. Akasaka, K. Bane, K. Egawa, A. Enomoto, J.W. Flanagan, Y. Funakoshi, K. Furukawa, K. Hayashi, S. Hiramatsu, H. Hisamatsu, K. Hosoyama, N. Huang, T. Ieiri, N. Iida, T. Kamitani, K. Kanazawa, S. Kato, M. Kikuchi, E. Kikutani, H. Koiso, T. Kubo, S. Kurokawa, T. Mitsuhashi, M. Masuzawa, T. Matsumoto, S. Michizono, T. Mimashi, T. Nakamura, Y. Ogawa, K. Ohmi, Y. Ohnishi, S. Ohsawa, N. Ohuchi, K. Oide, E. A. Perevedentsev, D. Pestrikov, K. Satoh, M. Suetake, Y. Suetsugu, T. Suwada, F. Takasaki, M. Tanaka, M. Tawada, M. Tejima, M. Tobiyama, T. Tsuboyama, N. Yamamoto, M. Yoshida, S. Yoshimoto, C. H. Yu, F. Zimmermann, and S. Olsen, in Proceedings of the European Particle Accelerator Conference (EPAC2000), Vienna, 2000 (European Physical Society, Geneva, 2000), p. 1122.

[7] A. Kulikov, A. S. Fisher, S. Heifets, J. T. Seeman, M. Sullivan, U. Wienands, and W. Kozanecki, in Proceedings of the Particle Accelerator Conference (PAC01), Chicago, IL, 2001 (Ref. [3]), p. 1903.

[8] Y. D. Liu, Z. Y. Guo, Q. Qin, J. Q. Wang, and J. Xing, in Proceedings of the Asian Particle Accelerator Conference (APAC04), Gyeongju, Korea, 2004 (Ref. [4]).

[9] M. A. Furman and G. R. Lambertson, LBNL Report No. LBNL-41123, 1997.

[10] M. Bassetti and G. A. Erskine, CERN Report No. CERN ISR Th/80-06, 1980.

[11] K. Ohmi and F. Zimmermann, Phys. Rev. Lett. 85, 3821 (2000).

[12] A. Chao, Physics of Collective Instability in High Energy Accelerators (Wiley, New York, 1993). 